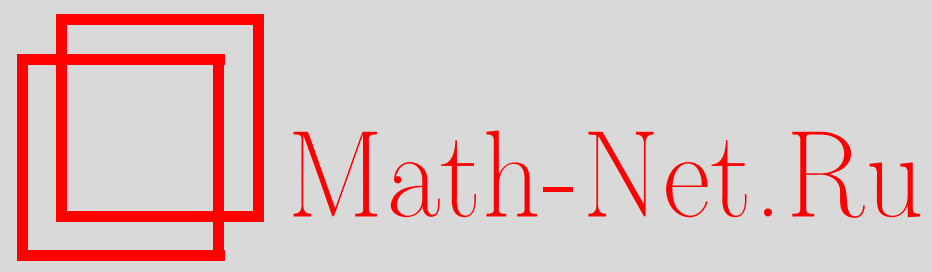

М. А. Алехина, О надежности схем в произвольном полном конечном базисе при однотипных константных неисправностях на выходах элементов, Дискрет. матем., 2012, том 24, выпуск 3, 17-24

DOI: https://doi.org/10.4213/dm1193

Использование Общероссийского математического портала Math-Net.Ru подразумевает, что вы прочитали и согласны с пользовательским соглашением http://www.mathnet.ru/rus/agreement

Параметры загрузки:

IP : 34.229 .108 .108

26 апреля 2023 г., 12:51:37

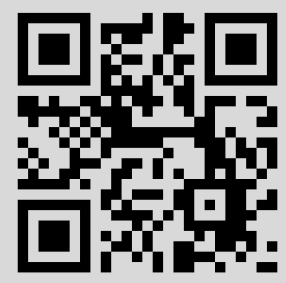




\title{
О надежности схем в произвольном полном конечном базисе при однотипных константных неисправностях на выходах элементов
}

\author{
() 2012 г. М. А. Алехина
}

\begin{abstract}
Рассматривается реализация булевых функций схемами из ненадежных функциональных элементов в произвольном полном конечном базисе $B$. Предполагается, что все элементы схемы независимо друг от друга с вероятностью $\gamma \in(0,1 / 2)$ подвержены однотипным константным неисправностям на выходах.

Доказано, что в базисе $B$ все булевы функции можно реализовать схемами, ненадежность которых не больше $3 \gamma+27 \gamma^{2}$ пр всех $\gamma \in(0,1 / 960)$.
\end{abstract}

Рассматривается реализация булевых функций схемами из ненадежных функциональных элементов в произвольном полном конечном базисе $B$. Будем считать, что схема из ненадежных функциональных элементов реализует функцию $f\left(x_{1}, \ldots, v_{n}\right)$, если при поступлении на входы схемы набора $\tilde{A}=\left(a_{1}, \ldots, a_{n}\right)$ при отсутствии неисправностей на выходе схемы появляется значение $f(\tilde{a})$. Все элементы схемы независимо друг от друга с вероятностью $\gamma \in(0,1 / 2)$ переходят в неисправное состояние типа 0 на выходах элементов. Эти неисправности характеризуются тем, что в исправном состоянии функциональный элемент реализует приписанную ему булева функцию $\varphi$, а в неисправном состоянии константу 0. Неисправности типа 1 на выходах элементов определяются аналогично.

Всюду далее, исключая теорему 2, считаем, что базисные элементы подвержены неисправностям типа 0 на выходах.

Пусть $P_{\bar{f}(\tilde{a})}(S, \tilde{a})$ - вероятность появления значения $\bar{f}(\tilde{a})$ на выходе схемы $S$, реализующей функцию $f(\tilde{x})$ при наборе $\tilde{a}$. Ненадежность $P(S)$ схемы $S$ определяется как $\max \left\{P_{\bar{f}(\tilde{a})}(S, \tilde{a})\right\}$, где максимум берется по всем наборам $\tilde{a}$. Надежность схемы $S$ равна $1-P(S)$.

Пусть

$$
P_{\gamma}(f)=\inf P(S),
$$

где $S$ - схема, реализующая $f(\tilde{x})$. Схему $A$, реализующую $f$, назовем асимптотически оптимальной по надежности, если $P(A) \sim P_{\gamma}(f)$ при $\gamma \rightarrow \infty$.

Нетрудно проверить, что ненадежность любой схемы, реализующей неконстантную функцию и содержащей хотя бы один функциональный элемент, не меньший $\gamma$.

Очевидно, что функции $x_{i}, i \in\{1, \ldots, n\}$, можно реализовать абсолютно надежно (не используя функциональных элементов), а функции, для реализации которых достаточно $k$ элементов, $k \in\{1,2,3\}$, с ненадежностью, не большей $k \gamma, \gamma \in(0,1 / 2)$. Для любой функции в произвольном полном конечном базисе справедлива следующая теорема. 
Теорема 1. В произвольном полном конечном базисе любую булеву функцию $f$ можно реализовать такой схемой $A$, что при всех $\gamma \in(0,1 / 960]$ верно неравенство

$$
P(A) \leqslant 3 \gamma+27 \gamma^{2} \text {. }
$$

Для инверсных неисправностей на выходах элементов, которые характеризуются тем, что в исправном состоянии функциональный элемент реализует приписанную ему булеву функцию $\varphi$, а в неисправном - функцию $\bar{\varphi}$, справедлива следующая теорема.

Теорема 2 ([1]). Пусть В - произвольный полный конечный базис, а базисныле элементыс с вероятностью є подвержены инверсным неисправностям на выходах. Тогда существуют положительные константы $\varepsilon_{0}$ и с такие, что любую функиию $f$ можно реализовать схемой $S$ над $B$, для которой при всех $\varepsilon \in\left(0, \varepsilon_{0}\right]$ справедливо неравенство

$$
P(S) \leqslant 5 \varepsilon+c \varepsilon^{2} .
$$

Идея доказательства теоремы 1 такая же, как и теоремы 2, однако некоторая модификация позволила сократить перебор возможных случаев и объем доказательства.

Чтобы доказать теорему 1, сформулируем необходимые вспомогательные утверждения.

Теорема 3 ([2]). В полном базисе из двухвходовых функииональных элементов любую булеву функцию $f$ можно реализовать такой схемой $S$, что при всех $\gamma \in(0,1 / 480]$ верно неравенство

$$
P(S) \leqslant 3 \gamma+27 \gamma^{2}
$$

Обозначим через $G$ множество функций, зависящих от переменных $x_{1}, x_{2}, x_{3}$ и конгруэнтных одной из функций

$$
g\left(x_{1}, x_{2}, x_{3}\right)=x_{1}^{c_{1}} x_{2}^{c_{2}} \vee x_{1}^{c_{1}} x_{3}^{c_{3}} \vee x_{2}^{c_{2}} x_{3}^{c_{3}}, \quad c_{i} \in\{0,1\}, \quad i=1,2,3 .
$$

Лемма 1 ([2]). Допустим, что произвольную функцию $f$ можно реализовать схемой $S c$ ненадежностью, не большей $p$. Пусть $S_{g}-$ схема, реализующая некоторую функцию $g$ из множества $G$ с ненадежностью, не большей $P\left(S_{g}\right)$, причем $v_{0}$ - вероятность очибки схемь $S_{g}$ на наборе $\left(c_{1}, c_{2}, c_{3}\right)$, а $v_{1}-$ на наборе $\left(\bar{c}_{1}, \bar{c}_{2}, \bar{c}_{3}\right)$. Тогда можно построить схему $\Phi(S)$, которая реализует функцию $f$ с ненадежностью

$$
P\left(\Phi(S) \leqslant \max \left\{v_{0}, v_{1}\right\}+3 p P\left(S_{g}\right)+3 p^{2} .\right.
$$

Лемма 2 ([3]). В произвольном полном конечном базисе любую булеву функцию $f$ можно реализовать такой схемой $D$, что при всех $\gamma \in(0,1 / 960]$ справедливо неравенство

$$
P(D) \leqslant 5 \gamma+182 \gamma^{2} \leqslant 5,2 \gamma .
$$

Обозначим через $T_{0}$ множество функций, сохраняющих константу 0 , через $T_{1}-$ множество функций, сохраняющих константу 1 , через $L-$ множество линейных функций, и через $M$ обозначим множество монотонных функций.

Лемма 3 ([6]). Пусть $f_{0}\left(x_{1}, \ldots, x_{k}\right) \notin T_{0} u f\left(x_{1}, \ldots, x_{m}\right) \notin T_{1}$. Тогда либо $\bar{x} \in$ $\left\{f_{0}(x, \ldots, x), f_{1}(x, \ldots, x)\right\}$, либо $f_{0}(x, \ldots, x) \equiv 1, f_{1}(x, \ldots, x) \equiv 0$.

Булевы функции $x_{1} x_{2} \oplus x_{1} x_{3} \oplus x_{2} x_{3} \oplus a_{1} x_{1} \oplus a_{2} x_{2} \oplus a_{3} x_{3} \oplus a_{0}, a_{i} \in\{0,1\}, i \in\{0,1,2,3\}$, будем называть особенными [5]. 
Лемма 4 ([5]). Из всякой нелинейной и неособенной функции трех или более переменньх, подстановкой переменных или введением фиктивных переменных можно получить либо особенную функцию, либо нелинейную функиию двух переменных.

Из леммы 4 следует, что для всякой нелинейной функции $f_{L}$ имеет место ровно один из вариантов: либо $f_{L}$ является особенной функцией, либо $f_{L}-$ функция двух переменных, либо из неособенной функции $f_{L}\left(x_{1} m \ldots, x_{n}\right), n \geqslant 3$, подстановкой переменных [6] можно получить особенную функцию, либо из неособенной функции $f_{L}\left(x_{1}, \ldots, x_{n}\right)$, $n \geqslant 3$, отождествлением переменных можно получить нелинейную функцию двух переменных. Таким образом, получаем очевидное следствие.

Следствие 1. Из всякой нелинейной функции $f_{L}$ подстановкой переменных можно получить функцию, равную либо некоторой особенной функиии

$$
\varphi\left(x_{1}, x_{2}, x_{3}\right)=x_{1} x_{2} \oplus x_{1} x_{3} \oplus x_{2} x_{3} \oplus a_{0},
$$

либо некоторой нелинейной функичии двух переменных

$$
\psi\left(x_{1}, x_{2}\right)=x_{1} x_{2} \oplus a_{1} x_{1} \oplus a_{2} x_{2} \oplus a_{0},
$$

где $a_{i} \in\{0,1\}, i=0,1,2,3$.

Лемма 5 ([1]). Пусть $f\left(x_{1}, \ldots, x_{n}\right) \notin M, n \geqslant 3$. Тогда из $f$ подстановкой переменных $u$ введением фиктивных переменных можно получить такую функцию $\varphi\left(x_{1}, x_{2}, x_{3}\right) \notin M$, чтоо

$$
\varphi(x, 0,1)=\bar{x}
$$

Доказательство. Доказательство этой леммы отличается от доказательства, приведенного в [1]. Пусть $f\left(x_{1}, \ldots, x_{n}\right) \notin M, n \geqslant 3$. Тогда (см. [6]) найдется $i \in\{1,2, \ldots, n\}$ и два соседние по $i$-й компоненте набора

$$
\begin{aligned}
& \tilde{\alpha}=\left(\alpha_{1}, \ldots, \alpha_{i-1}, 0, \alpha_{i+1} \ldots, \alpha_{n}\right), \\
& \tilde{\beta}=\left(\alpha_{1}, \ldots, \alpha_{i-1}, 1, \alpha_{i+1} \ldots, \alpha_{n}\right)
\end{aligned}
$$

такие, что

$$
f(\tilde{\alpha})=1, \quad f(\tilde{\beta})=0 .
$$

Перенумеруем переменные $x_{1}, \ldots, x_{n}$ следующим образом:

(1) переменную $x_{i}$ назовем $x_{1}$;

(2) переменную $x_{j}, j \in\{1, \ldots n\}, j \neq i$, назовем переменной $x_{2}$ тогда и только тогда, когда $\alpha_{j}=0$;

(3) переменную $x_{k}, k \in\{1, \ldots\},, k \neq i$, назовем переменной $x_{3}$ тогда и только тогда, когда $\alpha_{k}=1$.

В результате из функции $f$ получим такую функцию $\varphi\left(x_{1}, x_{2}, x_{3}\right) \notin M$, что

$$
\varphi(x, 0,1)=\bar{x} .
$$

Лемма 5 доказана. 
Замечание 1. Переменная $x_{i}, i=2,3$, для функции $\varphi\left(x_{1}, x_{2}, x_{3}\right)$ из леммы 5 может быть как существенной, так и фиктивной.

Замечание 2. Отличие леммы 5 от известной леммы о немонотонной функции [6] состоит в том, что используется только подстановка переменных, которая не влияет на вероятности ошибок функционального элемента.

Введем обозначения

$$
\begin{aligned}
& M_{0}=\left\{\bar{x}_{1} \bar{x}_{2}, \bar{x}_{1} \vee x_{2}, x_{1} \oplus x_{2} \oplus 1\right\}, \\
& M_{1}=\left\{\bar{x}_{1} \vee \bar{x}_{3}, \bar{x}_{1} x_{3}, x_{1} \oplus x_{3}\right\} .
\end{aligned}
$$

Лемма 6. Пусть $\varphi\left(x_{1}, x_{2}, x_{3}\right) \notin M u \varphi(x, 0,1)=\bar{x}$.

(1) Если $\varphi\left(x_{1}, x_{2}, x_{3}\right)$ сущуественно зависит только от переменной $x_{1}$, то

$$
\varphi\left(x_{1}, x_{2}, x_{3}\right)=\bar{x}_{1} .
$$

(2) Если $\varphi\left(x_{1}, x_{2}, x_{3}\right)$ существенно зависит только от переменных $x_{1}, x_{2}$, то

$$
\varphi\left(x_{1}, x_{2}, x_{3}\right) \in M_{0} .
$$

(3) Если $\varphi\left(x_{1}, x_{2}, x_{3}\right)$ сущчественно зависит только от переменных $x_{1}, x_{3}$, то

$$
\varphi\left(x_{1}, x_{2}, x_{3}\right) \in M_{1} .
$$

(4) Если $\varphi\left(x_{1}, x_{2}, x_{3}\right)$ существенно зависит от всех трех переменных $x_{1}, x_{2}, x_{3}$, то

$$
\varphi\left(x_{1}, 0, x_{3}\right) \in M_{1} \cup\left\{\bar{x}_{1}\right\} .
$$

Доказательство. Первое утверждение очевидным образом следует из условий леммы.

Докажем второе утверждение. Поскольку функция $\varphi\left(x_{!}, x_{2}, x_{3}\right)$ существенно зависит только от двух переменных $x_{1}, x_{2}$, представим ее многочленом:

$$
\varphi\left(x_{1}, x_{2}, x_{3}\right)=a_{1} x_{1} x_{2} \oplus a_{2} x_{1} \oplus a_{3} x_{2} \oplus a_{4} .
$$

По условию, $\varphi(x, 0,1)=\bar{x}$, следовательно, выполняются два равенства $a_{2}=1, a_{4}=1$. Получаем четыре решения:

$$
\begin{aligned}
& a_{1}=1, \quad a_{2}=1, \quad a_{3}=1, \quad a_{4}=1 ; \quad a_{1}=0, \quad a_{2}=1, \quad a_{3}=1, \quad a_{4}=1 ; \\
& a_{1}=1, \quad a_{2}=1, \quad a_{3}=0, \quad a_{4}=1 ; \quad a_{1}=0, \quad a_{2}=1, \quad a_{3}=0, \quad a_{4}=1 \text {. }
\end{aligned}
$$

Каждому из этих решений соответствует одна из функций $\bar{x}_{1} \bar{x}_{2}, \bar{x}_{1} \vee x_{2}, x_{1} \oplus x_{2} \oplus 1, \bar{x}_{1}$. Переменные $x_{1}, x_{2}$ - существенные для $\varphi\left(x_{1}, x_{2}, x_{3}\right)$, поэтому $\varphi\left(x_{1}, x_{2}, x_{3}\right) \in M_{0}$.

Докажем третье утверждение. Поскольку функция $\varphi\left(x_{1}, x_{2}, x_{3}\right)$ существенно зависит только от двух переменных $x_{1}, x_{3}$, представим ее многочленом:

$$
\varphi\left(x_{1}, x_{2}, x_{3}\right)=a_{1} x_{1} x_{3} \oplus a_{2} x_{1} \oplus a_{3} x_{3} \oplus a_{4} .
$$

По условию, $\varphi(x, 0,1)=\bar{x}$, следовательно, выполняются два равенства $a_{1} \oplus a_{2}=1$, $a_{3} \oplus a_{4}=1$. Решая два последних уравнения, получим четыре решения:

$$
\begin{aligned}
& a_{1}=0, \quad a_{2}=1, \quad a_{3}=1, \quad a_{4}=0 ; \quad a_{1}=0, \quad a_{2}=1, \quad a_{3}=0, \quad a_{4}=1 ; \\
& a_{1}=1, \quad a_{2}=0, \quad a_{3}=1, \quad a_{4}=0 ; \quad a_{1}=1, \quad a_{2}=0, \quad a_{3}=0, \quad a_{4}=1 \text {. }
\end{aligned}
$$


Каждому из этих решений соответствует одна из функций $\bar{x}_{1}, x_{1} x_{3} \oplus 1, \bar{x}_{1} x_{3}, x_{1} \oplus x_{3}$. Переменные $x_{1}, x_{3}$ - существенные для $\varphi\left(x_{1}, x_{2}, x_{3}\right)$, поэтому $\varphi\left(x_{1}, x_{2}, x_{3}\right) \in M_{1}$.

Докажем четвертое утверждение. Представим функцию $\varphi\left(x_{1}, 0, x_{3}\right)$ многочленом

$$
\varphi\left(x_{1}, 0, x_{3}\right)=a_{1} x_{1} x_{3} \oplus a_{2} x_{1} \oplus a_{3} x_{3} \oplus a_{4}
$$

и рассуждая, как и в предыдущем пункте, получим, что $\varphi\left(x_{1}, 0, x_{3}\right) \in M_{1} \cup\{\bar{x}\}$.

Лемма 6 доказана.

Замечание 3. Леммы 5 и 6 позволят в дальнейшем от произвольной немонотонной функции подстановкой переменных перейти сразу к немонотонной функции зависящей не более, чем от двух переменных, или же получить такую функцию, применив еще и подстановку константы 0. Поскольку функциональный элемент, реализующий константу 0 , абсолютно надежен при неисправностях типа 0 на выходах, его использование не влияет на вероятности ошибок исходной схемы. В то же время, подстановка константы 1 нежелательна, поскольку функциональный элемент, реализующий константу 1 , ненадежен при рассматриваемых неисправностях, и его использование увеличит ненадежность исходной схемы.

Доказательство теоремы 1. Пусть $B$ - произвольный полный конечный базис, а $f-$ любая булева функция. Поскольку базис $B$ полный, в нем содержится нелинейная функция $f_{L}$. Из функции $f_{L}$ (см. лемму 4 и следствие 1 ) подстановкой переменных можно получить функцию, равную либо

$$
\varphi\left(x_{1}, x_{2}, x_{3}\right)=x_{1} x_{2} \oplus x_{1} x_{3} \oplus x_{2} x_{3} \oplus a_{1} x_{1} \oplus a_{2} x_{2} \oplus a_{3} x_{3} \oplus a_{0},
$$

либо

$$
\psi\left(x_{1}, x_{2}\right)=x_{1} x_{2} \oplus a_{1} x_{1} \oplus a_{2} x_{2} \oplus a_{0},
$$

где $a_{i} \in\{0,1\}, i=0,1,2,3$.

1. Пусть

$$
\varphi\left(x_{1}, x_{2}, x_{3}\right)=x_{1} x_{2} \oplus x_{1} x_{3} \oplus x_{2} x_{3} \oplus a_{1} x_{1} \oplus a_{2} x_{2} \oplus a_{3} x_{3} \oplus a_{0} .
$$

По лемме 2, функцию $f$ можно реализовать такой схемой $D$, что при всех $\gamma \in$ $(0,1 / 960]$ верно неравенство

$$
P(D) \leqslant 5,2 \gamma
$$

1.1. Пусть функция $\varphi\left(x_{1}, x_{2}, x_{3}\right)$ конгруэнтна функции

$$
\varphi_{1}\left(x_{1}, x_{2}, x_{3}\right)=x_{1} x_{2} \oplus x_{1} x_{3} \oplus x_{2} x_{3} \oplus a_{4}\left(x_{2} \oplus x_{3}\right) \oplus a_{0} .
$$

Тогда

$$
\varphi_{1}\left(x_{1}, x_{2}, x_{3}\right)=x_{1}^{\bar{a}_{0} \oplus a_{4}} x_{2}^{\bar{a}_{0}} \oplus x_{1}^{\bar{a}_{0} \oplus a_{4}} x_{3}^{\bar{a}_{0}} \oplus x_{2}^{\bar{a}_{0}} x_{3}^{\bar{a}_{0}},
$$

иными словами,

$$
\varphi_{1}\left(x_{1}, x_{2}, x_{3}\right)=g\left(x_{1}, x_{2}, x_{3}\right) \in G .
$$


Таким образом, для реализации функции $g$ из множества $G$ достаточно только одного функционального элемента $E_{g}$, поэтому

$$
v_{1}=0, \quad v_{0}=P\left(E_{g}\right)=\gamma .
$$

Применяя лемму 1 , по схеме $D$ с использованием элемента $E_{g}$ построим такую схему $\Phi_{1}(D)$, реализующую функцию $f$, что

$$
P\left(\Phi_{1}(D)\right) \leqslant \gamma+3 \cdot 5,2 \gamma \cdot \gamma+3(5,2 \gamma)^{2} \leqslant \gamma+97 \gamma^{2}<2 \gamma
$$

Схема $\Phi_{1}(D)=A-$ искомая.

1.2. Пусть функция $\varphi\left(x_{1}, x_{2}, x_{3}\right)$ конгруэнтна функции

$$
\varphi_{2}\left(x_{1}, x_{2}, x_{3}\right)=x_{1} x_{2} \oplus x_{1} x_{3} \oplus x_{2} x_{3} \oplus x_{3} \oplus a_{4}\left(x_{1} \oplus x_{2}\right) \oplus a_{0} .
$$

Отождествим переменные $x_{1}$ и $x_{2}$, и получим функцию

$$
\varphi_{2}\left(x_{1}, x_{2}, x_{3}\right)=x_{1} \oplus x_{3} \oplus a_{0} .
$$

Реализуя формулу $\varphi_{2}\left(\varphi_{2}\left(x_{1}, x_{2}, x_{3}\right), \varphi_{2}\left(x_{1}, x_{2}, x_{3}\right), x_{3}\right)$, построим схему $S_{g}$ из двух элементов, реализующую некоторую функцию $g$ из множества $G$ :

$$
\begin{aligned}
g\left(x_{1}, x_{2}, x_{3}\right) & =\varphi_{2}\left(\varphi_{2}\left(x_{1}, x_{2}, x_{3}\right), \varphi_{2}\left(x_{1}, x_{2}, x_{3}\right), x_{3}\right) \\
& =x_{1} x_{2} \oplus x_{1} x_{3} \oplus x_{2} x_{3} \oplus a_{4}\left(x_{1} \oplus x_{2}\right) .
\end{aligned}
$$

Тогда, в силу леммы $1, v_{0}, v_{1}, P\left(S_{g}\right) \leqslant 2 \gamma$.

Используем лемму 1 : возьмем схему $D$, и с помощью схемы $S_{g}$ построим схему $\Phi(D)$, реализующую функцию $f$, такую, что

$$
P(\Phi(D)) \leqslant 2 \gamma+3 \cdot 5,2 \gamma \cdot 2 \gamma+3(5,2 \gamma)^{2} \leqslant 2 \gamma+113 \gamma^{2} .
$$

Воспользуемся леммой 1 еще раз: по схеме $\Phi(D)$ с использованием схемы $S_{g}$ построим такую схему $\Phi^{2}(D)$, реализующую функцию $f$, что

$$
P\left(\Phi^{2}(D)\right) \leqslant 2 \gamma+3\left(2 \gamma+113 \gamma^{2}\right) 2 \gamma+3\left(2 \gamma+113 \gamma^{2}\right)^{2} \leqslant 2 \gamma+27 \gamma^{2}
$$

при всех $\gamma \in(0,1 / 960]$. Схема $\Phi^{2}(D)=A-$ искомая.

2. Пусть

$$
\psi\left(x_{1}, x_{2}\right)=x_{1} x_{2} \oplus a_{1} x_{1} \oplus a_{2} x_{2} \oplus a_{0},
$$

то есть функция $\psi\left(x_{1}, x_{2}\right)$ конгруэнтна одной из функций $\bar{x}_{1} \bar{x}_{2}, \bar{x}_{1} \vee \bar{x}_{2}, x_{1} x_{2}, x_{1} \vee x_{2}$, $\bar{x}_{1} x_{2}, \bar{x}_{1} \vee x_{2}$.

Если базис $B$ содержит хотя бы одну из функций $\bar{x}_{1} \bar{x}_{2}, \bar{x}_{1} \vee \bar{x}_{2}$, по теореме 3 утверждение верно. Поэтому будем рассматривать только случаи, когда $\psi\left(x_{1}, x_{2}\right)$ конгруэнтна одной из функций $x_{1} x_{2}, x_{1} \vee x_{2}, \bar{x}_{1} x_{2}, \bar{x}_{1} \vee x_{2}$.

По условию базис $B$ полный, поэтому он содержит функции $f_{0}\left(x_{1}, \ldots, x_{k}\right) \notin T_{0}$ и $f_{1}\left(x_{1}, \ldots, x_{m}\right) \notin T_{1}$, из которых, по лемме 3 , отождествлением переменных можно получить либо $\bar{x} \in\left\{f_{0}(x, \ldots, x), f_{1}(x \ldots, x)\right\}$, либо $f_{0}(x, \ldots, x) \equiv 1, f_{1}(x, \ldots, x) \equiv 0$. 
2.1. Пусть $\bar{x} \in\left\{f_{0}(x, \ldots, x), f_{1}(x, \ldots, x)\right\}$. Тогда имеем 4 полных базиса $\left\{x_{1} x_{2}, \bar{x}_{1}\right\}$, $\left\{x_{1} \vee x_{2}, \bar{x}_{1}\right\},\left\{\bar{x}_{1} x_{2}, \bar{x}_{1}\right\},\left\{\bar{x}_{1} \vee x_{2}, \bar{x}_{1}\right\}$; для каждого из них по теореме 3 утверждение верно.

\section{2. Пусть}

$$
f_{0}(x, \ldots, x) \equiv 1, \quad f_{1}(x, \ldots, x) \equiv 0 .
$$

В полных базисах $\left\{\bar{x}_{1} x_{2}, 1\right\},\left\{\bar{x}_{2} \vee x_{2}, 0\right\}$ по теореме 3 утверждение верно. Поэтому далее будем считать, что нелинейная функция $\psi\left(x_{1}, x_{2}\right)$ равна $x_{1} x_{2}$ или $x_{1} \vee x_{2}$.

Базис $B$ полный, поэтому в нем содержится немонотонная функция $f_{M}$. Если функция $f_{M}$ зависит не более, чем от двух переменных, то она конгруэнтна одной из функций множества

$$
M_{2}=M_{0} \cup M_{1} \cup\left\{\bar{x}_{1}\right\}
$$

Добавим к каждому из множеств $\left\{x_{1} x_{2}, 0,1\right\},\left\{x_{1} \vee x_{2}, 0,1\right\}$ произвольную немонотонную функцию из $M_{2}$ и получим полный базис из двухвходовых элементов, к которому применима теорема 3. Следовательно, утверждение теоремы 1 верно.

Пусть функция $f_{M}$ зависит не менее, чем от трех переменных. Тогда, по лемме 5, из функции $f_{M}$ подстановкой переменных можно получить такую немонотонную функцию $\varphi\left(x_{1}, x_{2}, x_{3}\right)$, что $\varphi\left(x_{1}, 0,1\right)=\bar{x}_{1}$. По лемме 6 , или $\varphi\left(x_{1}, x_{2}, x_{3}\right) \in M_{2}$, или функция $\varphi\left(x_{1}, x_{2}, x_{3}\right)$ такова, что $\varphi\left(x_{1}, 0, x_{3}\right) \in M_{1} \cup\left\{x_{1}\right\}$. В первом случае утверждение теоремы 1 верно (см. рассуждения выше).

Пусть функция $\varphi\left(x_{1}, x_{2}, x_{3}\right)$ такова, что $\varphi\left(x_{1}, 0, x_{3}\right) \in M_{1} \cup\left\{\bar{x}_{1}\right\}$. Используя два функциональных элемента, один из которых реализует функцию $\varphi\left(x_{1}, x_{2}, x_{3}\right)$, а второй - константу 0 , и моделируя формулу $\varphi\left(x_{1}, 0, x_{3}\right)$, построим схему из двух элементов, которая реализует одну из функций множества $M_{1} \cup\left\{\bar{x}_{1}\right\}$ и функционирует с теми же вероятностями ошибок на соответствующих наборах, что и один функциональный элемент, подверженный с вероятностью $\gamma$ неисправности типа 0 на выходе (поскольку элемент, реализующий константу 0 , функционирует абсолютно надежно). Снова применим теорему 3, согласно которой утверждение верно.

Теорема 1 доказана.

Из теоремы 1 следует, что а произвольном полном конечном базисе при неисправностях типа 0 на выходах элементов любую булеву функцию можно реализовать схемой, ненадежность которой при всех $\gamma \in(0,1 / 960]$ не больше $3 \gamma+27 \gamma^{2}$.

Известно (см. [2]), что в общем случае константу 3 в оценке ненадежности понизить нельзя. Например, в базисах $\left\{x_{1} x_{2}, \bar{x}_{1}\right\},\left\{\bar{x}_{1} x_{2}, 1\right\}$ для почти всех функций асимптотически оптимальные по надежности схемы функционируют с ненадежностью, асимптотически равной $3 \gamma$ при $n \rightarrow \infty$. Кроме того, в базисе $\left\{\bar{x}_{1} \vee \bar{x}_{2}\right\}$ для почти всех функций асимптотически оптимальные по надежности схемы функционируют с ненадежностью, асимптотически равной $2 \gamma$, а в базисе $\left\{\bar{x}_{1} \bar{x}_{2}\right\}$ для почти всех функций асимптотически оптимальные по надежности схемы функционируют с ненадежностью, асимптотически равной $\gamma$ при $\gamma \rightarrow 0$.

Поскольку ненадежности двойственных схем равны (см. [4]), теорема 1 верна в произвольном полном конечном базисе при неисправностях типа 1 на выходах базисных элементов. 


\section{Список литературы}

1. Аксенов С. И., О надежности схем над произвольной полной системой функций при инверсных неисправностях на выходах элементов. Известия высших учебных заведений. Поволжский регион. Естественные науки (2005) 21, №6, 42-55.

2. Алехина М. А., Синтез асимптотически оптимальных по надежности схем. Информ.-издат. центр ПГУ, Пенза, 2006.

3. Алехина М. А., О надежности схем в базисах, содержащих медиану. В сб.: Tруды VIII международной конференции «Дискретные модели в теории управляющих систем». МАКС, Москва, 2009 , с. $13-17$.

4. Алехина М. А., Пичугина П. Г., О надежности двойственных схем в полном конечном базисе. В сб.: Материалы ХVIII Международной школь-семинара «Синтез и сложность управляюших систем». Мех.-мат. ф-т МГУ, Москва, 2009, с. 10-13.

5. Редькин Н. П., О полных проверяющих тестах. Математические вопросы кибернетики (1989) 2, 198-222.

6. Яблонский С. В., Введение в дискретную математику. Высшая школа, Москва, 2001.

Статья поступила 30.03.2011. 